\title{
Etude de la réactivation de la phosphatase alcaline dans le lait stérilisé par friction*
}

\author{
par \\ J. GED et C. ALAIS \\ Laboratoire de Biochimie Appliquée, \\ Université de Nancy-I,F-54037 Nancy
}

\section{INTRODUCTION}

La phosphatase alcaline, dont la présence dans le lait a été révélée en 1933 [1], a donné lieu à de très nombreux travaux qui sont, pour la plupart, en relation directe avec le contrôle de la pasteurisation industrielle. On sait que la courbe de dénaturation thermique de cet enzyme se situe un peu au-dessus de celle de la destruction de Mycobacterium tuberculosis, l'espèce pathogène non sporulée la plus thermorésistante dans le lait cru ; mais il a été montré que, dans certaines conditions de chauffage, l'activité phosphatasique peut réapparaître, indépendamment de toute croissance microbienne, ce qui montre que la dénaturation implique des changements de conformation réversibles, au moins partiellement.

Dans notre laboratoire, la fraction dominante de la phosphatase alcaline (E.C.3.1.3.1.) dans le lait a fait l'objet de récentes investigations $[2,3,4]$; c'est une métalloglycoprotéine à zinc et à magnésium structuraux; elle se trouve liée aux particules lipoprotidiques de la membrane des globules gras. L'existence de plusieurs formes moléculaires a été postulée ; il y en aurait trois selon Peereboom $[5,6]$ avec des poids moléculaires de 140000 et 570000 , dont une seule se réactiverait après chauffage, et deux selon Lefranc et Han [7], A et $B$, avec des poids moléculaires respectivement de 140000 et plus de 200 000. Pour Andrews [8] et Barman et Gutfreud [9] la phosphatase alcaline du lait de vache a un poids moléculaire de 180000 190000 . Nous avons purifié la phosphatase alcaline à partir du babeurre et ses caractéristiques moléculaires et cinétiques ont été étudiées ; son poids moléculaire est voisin de 170000 [2, 3].

$\div 4^{\mathrm{e}}$ communication sur les effets biochimiques du procédé de stérilisation ATAD. 
Dans le lait, la réactivation se manifeste surtout après des traitements de courte durée à température assez élevée : l'activité récupérée reste toujours faible, de l'ordre de 1 p. $100[10,11]$ alors que dans la crème ou dans des milieux simplifiés, elle peut être beaucoup plus importante $[10,12]$. Les facteurs de la réactivation, en dehors des conditions du chauffage, sont la vitesse du refroidissement, la température et la durée de conservation et l'acidité $[10,11]$.

Dans le cadre de notre étude sur les effets biochimiques de la stérilisation ultra-rapide par friction (procédé ATAD), nous nous sommes naturellement intéressés au devenir de la phosphatase alcaline dans diverses conditions de chauffage.

\section{MATERIEL ET METHODES}

Nous avons utilisé du lait de grand mélange stérilisé par le procédé ATAD (appareil de type UF 300). Rappelons qu'il consiste à chauffer le lait en le faisant passer entre un disque tournant à grande vitesse $(4000-5000 \mathrm{t} / \mathrm{mn})$ et deux flasques fixes ; en raison de l'écart très réduit $(0,3 \mathrm{~mm})$, le lait entrant au voisinage du moyeu et sortant à la périphérie subit un échauffement interne par friction extrêmement rapide $\left(0,3 \mathrm{~s}\right.$ à $130^{\circ} \mathrm{C}$ et $0,8 \mathrm{~s}$ à $\left.150^{\circ} \mathrm{C}\right)$. Le préchauffage à $70^{\circ} \mathrm{C}$, d'une durée de 4 à $6 \mathrm{~s}$, et le refroidissement du lait à $10^{\circ} \mathrm{C}$ sont assurés par des échangeurs appropriés.

La stérilisation à l'autoclave a été effectuée pendant $20 \mathrm{mn}$ à $115^{\circ} \mathrm{C}$. Le lait UHT a été prélevé à la sortie de l'appareil de conditionnement aseptique d'une usine ; le traitement comportait une pasteurisation à $72^{\circ} \mathrm{C}$ durant $15 \mathrm{~s}$, puis, après $24 \mathrm{~h}$, une stérilisation à $142^{\circ} \mathrm{C}$ pendant $2 \mathrm{~s}$.

Nous avons dosé l'activité de la phosphatase alcaline dans le lait cru et stérilisé à $130^{\circ} \mathrm{C}, 140^{\circ} \mathrm{C}, 150^{\circ} \mathrm{C}$ et $160^{\circ} \mathrm{C}$, au temps 0 et jusqu'à $72 \mathrm{~h}$ de conservation à $34^{\circ} \mathrm{C}$ qui est la température la plus favorable à la réactivation [11].

Nous avons utilisé la méthode d'Aschaffenburg and Mullen [13] qui consiste à faire agir l'enzyme du lait sur une solution de paranitrophényl-phosphate disodique pendant $2 \mathrm{~h}$ à $37^{\circ} \mathrm{C}$; on arrête la réaction par addition d'acide trichloracétique à $28 \mathrm{p} .100$; on filtre pour éliminer les protéines précipitées et on réalcalinise le filtrat avec un mélange de carbonate de sodium et d'héxamétaphosphate de sodium, avant la mesure spectrophotométrique à $410 \mathrm{~nm}$ du paranitrophénol (P.N.P.) libéré par l'enzyme. En se référant à une courbe d'étalonnage obtenue avec du lait bouilli et des quantités de P.N.P. allant de $0-100 \mu \mathrm{M}$ on peut transformer la densité optique en $\mu \mathrm{M}$ de P.N.P. 


\section{RESULTATS}

Le tableau 1 présente l'activité phosphatasique, en $\mu \mathrm{M}$ de P.N.P. dans le lait stérilisé. Les valeurs indiquées sont la moyenne de cinq séries d'essais pour les laits traités par le procédé ATAD. Nous avons observé une certaine dispersion des résultats, comme l'avaient constaté Sharma et Ganguli [11] dans le cas du lait chauffé à $80^{\circ} \mathrm{C}-100^{\circ} \mathrm{C}$ et Do Ngoc-Cu [12] dans le traitement de la crème à $90^{\circ} \mathrm{C}$; les coefficients de variation sont du même ordre.

\section{TABLEAU 1}

Activité phosphatasique du lait stérilisé (en $\mu \mathrm{M}$ de paranitrophénol)

\begin{tabular}{|c|c|c|c|c|c|}
\hline \multirow{2}{*}{ Stérilisation } & \multicolumn{5}{|c|}{ Durée de conservation à $34^{\circ} \mathrm{C}$} \\
\hline & 0 & $10 \mathrm{~h}$ & $24 \mathrm{~h}$ & $48 \mathrm{~h}$ & $72 \mathrm{~h}$ \\
\hline 1) $\mathrm{ATAD}^{*}$ & & & & & \\
\hline$-130^{\circ} \mathrm{C}$ & 0 & 76 & 132 & 201 & 220 \\
\hline$-140^{\circ} \mathrm{C}$ & 0 & 48 & 122 & 206 & 232 \\
\hline$-150^{\circ} \mathrm{C}$ & 0 & 17 & 63 & 97 & 121 \\
\hline$-160 \mathrm{C}$ & 0 & - & 25 & 25 & 32 \\
\hline 2) Autoclave (115 $\mathrm{C}, 20 \mathrm{mn})$ & 0 & 0 & 0 & 0 & 0 \\
\hline 3) UHT industriel $\left(142^{\circ} \mathrm{C}, 2 \mathrm{~s}\right)$ & 0 & 0 & 0 & 0 & 6,5 \\
\hline
\end{tabular}

Au temps 0 , on n'observe jamais d'activité ; il en va de même après $72 \mathrm{~h}$ pour le lait autoclavé. Dans le lait U.H.T. on a observé à deux reprises une très faible activité après $72 \mathrm{~h}$ seulement. Les résultats obtenus pour le lait stérilisé par friction montrent une réactivation progressive de $10 \mathrm{~h}$ à $72 \mathrm{~h}$, dépendant de la température de stérilisation ; les valeurs s'échelonnent finalement de 32 à $232 \mu \mathrm{M}$ 
de P.N.P. Dans le lait cru, la valeur moyenne est de $3500 \mu \mathrm{M}$, avec des valeurs extrêmes de 2800 et $5200 \mu \mathrm{M}$.

La figure 1 présente les courbes moyennes de réactivation en p. 100 de la valeur initiale. Les courbes se disposent les unes au-dessus des autres selon l'ordre décroissant de la température, sauf pour la dernière partie de la courbe à $130^{\circ} \mathrm{C}$. Il est à noter que la stérilité n'est pas toujours totale à cette dernière température ; dans quatre essais sur cinq, le lait avait une acidité développée après 3 j ; dans le cinquième essai, la valeur de réactivation (10,3 p. 100) était nettement plus élevée que la moyenne (6,3 p. 100).

A $160^{\circ} \mathrm{C}$, une valeur maximale est atteinte en $24 \mathrm{~h}$. A des températures plus basses, l'augmentation du pourcentage de réactivation est rapide jusqu'à $48 \mathrm{~h}$; elle se ralentit ensuite ; les valeurs après $72 \mathrm{~h}$ doivent être près d'un maximum ; dans quelques expériences on

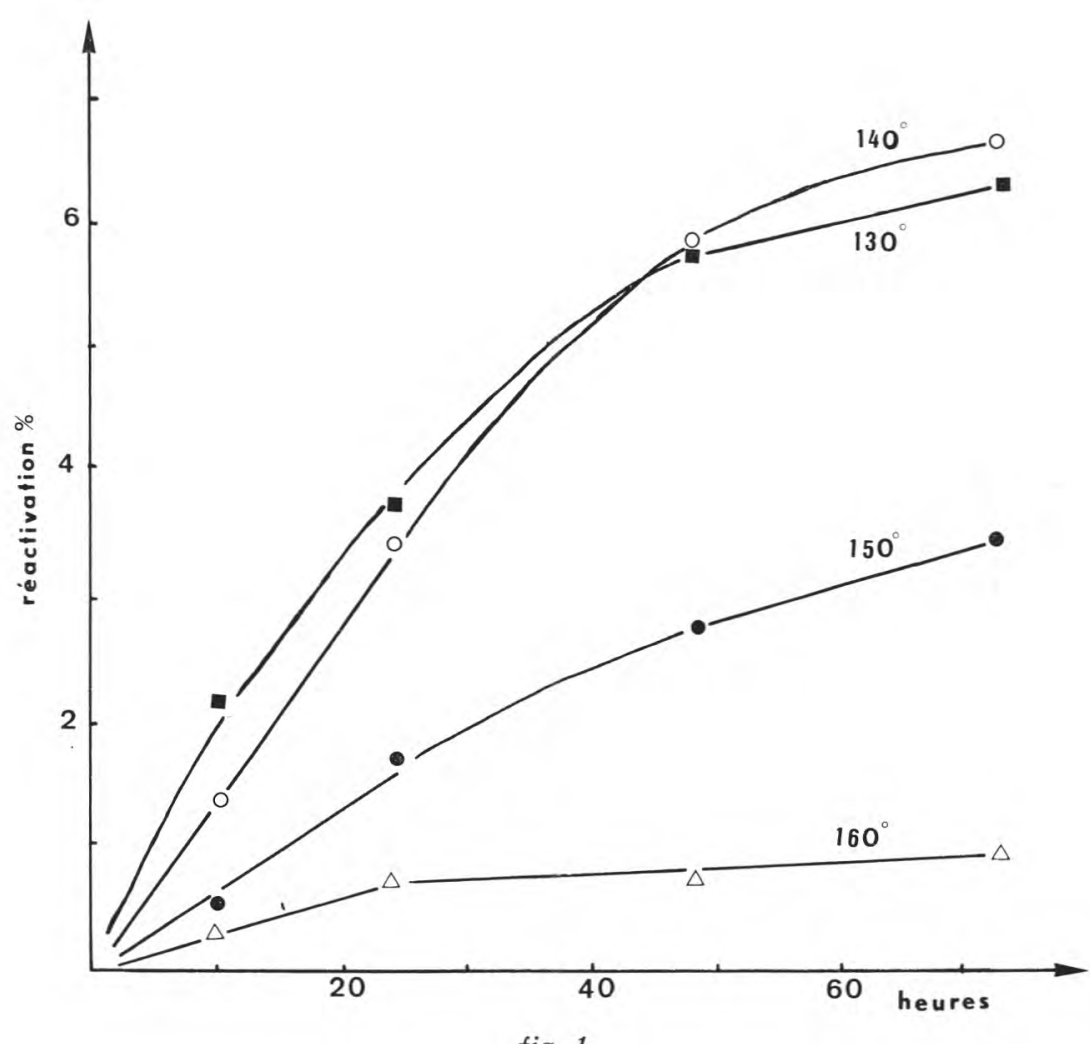

fig. 1

Réactivation de la phosphatase alcaline dans le lait stérilisé par friction, à différentes températures, en fonction de la durée de conservation à $34^{\circ} \mathrm{C}$. 
a mesuré l'activité après 96 h ; elle était égale ou un peu inférieure à celle mesurée après $72 \mathrm{~h}$.

\section{DISCUSSION}

La réactivation de la phosphatase alcaline dans le lait a été mesurée par différents auteurs à des températures de $80^{\circ} \mathrm{C}$ à $100^{\circ} \mathrm{C}$ (dans les conditions de la pasteurisation HTST on n'observe pas de réactivation). Les pourcentages de réactivation sont en général de l'ordre de 1 p. $100[10,11]$.

Le traitement par le procédé ATAD, dans des conditions assurant la stérilisation, permet des réactivations relativement importantes, de l'ordre de 3,5 p. 100 à $150^{\circ} \mathrm{C}$, et de 6,6 p. 100 à $140^{\circ} \mathrm{C}$. Ces températures sont supérieures à celles mentionnées ci-dessus, mais les durées du chauffage sont plus brèves, inférieures dans tous les cas à 1 s. Nos résultats montrent qu'un chauffage réellement instantané, à une température voisine de $140^{\circ} \mathrm{C}$ provoque une réactivation notable. A $160^{\circ} \mathrm{C}$, on observe encore une réactivation, mais inférieure à 1 p. 100. Le traitement UHT industriel (pasteurisation $72^{\circ} \mathrm{C} / 15 \mathrm{~s}$, stérilisation $142^{\circ} \mathrm{C} / 2 \mathrm{~s}$ ) ne provoque qu'une réactivation extrêmement lente et limitée ; l'enzyme a subi une dénaturation trop profonde, comme dans le cas de la stérilisation classique.

En ce qui concerne l'influence de la durée de conservation à $34^{\circ} \mathrm{C}$, un maximum de réactivation doit se présenter entre $72 \mathrm{~h}$ et $96 \mathrm{~h}$ pour le traitement $\mathrm{ATAD}$ à $130^{\circ} \mathrm{C}$ et $140^{\circ} \mathrm{C}$. Cette durée est beaucoup plus longue que celle indiquée par Sharma et Ganguli qui est de $10 \mathrm{~h}$ [11]; ces auteurs ne donnent pas un temps exact de chauffage mais indiquent que le lait est mis dans une fiole conique au bain-marie jusqu'à ce que la température de $90^{\circ} \mathrm{C}$ ou $100^{\circ} \mathrm{C}$ soit atteinte ; ces conditions sont probablement plus dénaturantes que celles du procédé par friction, même à la température de $160^{\circ} \mathrm{C}$.

Jusqu'à $48 \mathrm{~h}$ de conservation, la vitesse de réactivation est d'autant plus grande que la température est plus basse, entre $130^{\circ} \mathrm{C}$ et $160^{\circ} \mathrm{C}$; mais après $48 \mathrm{~h}$ le pourcentage de réactivation est plus haut à $140^{\circ} \mathrm{C}$ qu'à $130^{\circ} \mathrm{C}$. Ce fait est probablement la conséquence d'une acidification se développant lentement dans certains échantillons obtenus à $130^{\circ} \mathrm{C}$, température qui ne garantit pas la stérilité dans tous les cas ; on sait que l'acidité a un effet inhibiteur sur l'activité phosphatasique $[12,14]$.

La réactivation de la phosphatase alcaline reste un phénomène mal expliqué. Les hypothèses sont nombreuses. Nous poursuivons l'étude de cet enzyme sur le plan fondamental, en vue d'élucider ses transformations réversibles. 


\section{Remerciements}

Ces recherches ont été effectuées avec l'aide de la D.G.R.S.T. (contrats $n^{\circ}$ 73.7.1398 et 75.7.0383). Nous remercions la Société SEFFAC pour la mise à disposition du stérilisateur ATAD. Nous sommes reconnaissants à $M$. G. Linden pour ses conseils.

\section{Rés u m é}

Le procédé de stérilisation par friction (ATAD) produit un lait stérile dans lequel la phosphatase alcaline subit une réactivation notable au cours de la conservation à $34^{\circ} \mathrm{C}$; le maximum est atteint entre 3 et 4 j. L'enzyme est probablement moins dénaturé par le chauffage que dans d'autres modes de pasteurisation ou de stérilisation.

\section{S u m m a ry}

\section{REACTIVATION OF ALKALINE PHOSPHATASE IN MILK STERILIZED BY FRICTION}

The ATAD process gives a sterile milk in which alkaline phosphatase become rather strongly reactivated during conservation at $34^{\circ} \mathrm{C}$; the maximum value $\left(6,6 \mathrm{p} .100\right.$ at $\left.140 \mathrm{C}^{\circ}\right)$ is reached between 3 and 4 days. The enzyme is probably less denaturated by heating than in another pasteurization or sterilization process.

Reçu pour publication en avril 1976.

\section{Bibliographie}

[1] Graham (W. R.) and Kay (H. D.) (1933). - Phosphorus compounds of milk. V. The phosphorus partition in milk with preliminary observations on milk phosphatase. J. Dairy Res., 5, 54-63.

[2] Linden (G.), Mazeron (P.), Michalowski (J. B.) et Alais (C.) (1974). - Phosphatase alcaline du lait de vache. I. Purification et propriétés moléculaires. Biochim. Biophys. Acta, 358, 82-90.

[3] Linden (G.) et Alais (C.) (1976). - Phosphatase alcaline du lait. II. Structure sous-unitaire, nature métallo-protéique et paramètres cinétiques. Biochim. Biophys. Acta, 429, 205-213.

[4] LORIENT (D.) and Linden (G.) (1976). - Dephosphorylation of cows casein by milk alkaline phosphatase. J. Dairy Res., 43, 19-26.

[5] Peereboom (J. W. C.) (1966). - Studies on alkaline milk phosphatase. I. Non identity of raw and reactivated alkaline phosphatases from cream. Neth. Milk. Dairy J., 20, 113-122. 
[6] Peereboom (J. W. C.) (1968). - Studies on alkaline milk phosphatase. II. Occurence of various phosphatase isozymes in dairy products. Neth. Milk Dairy J., 22, 137-152.

[7] Lefranc (G.) et Han (K.) (1967). - Isolement et purification de deux fractions de la phosphatase alcaline du lait de vache. Ann. Institut Pasteur, Lille, 18, 185-196.

[8] ANDREwS (P.) (1965). - The Gel-Filtration behaviour of proteins related to their molecular weights over a wide range. Biochem. J., 96, 596-606.

[9] BARMAN (T. E.) and GutFreud (H.) (1966). - The catalytic center activity and kinetic properties of bovine milk alkaline phosphatase. Biochem. J., 101, 460-466.

[10] Lyster (R. L.) and Aschaffenburg (R.) (1962). - The reactivation of milk alkaline phosphatase after heat treatment. J. Dairy Res., 29, 21-35.

[11] Sharma (R. S.) and Ganguli (M. C.) (1974). - Some observations on the reactivation of alkaline phosphatase of buffalo milk. Milchwissenschaft, 29, 408-411.

[12] Do Ngoc-Cu, Lenoir (J.) et Veisseyre (R.) (1965). - Contribution à l'étude des conditions de la réactivation de la phosphatase alcaline dans les crèmes pasteurisées. Le Lait, 45, 387-411.

[13] Aschaffenburg (R.) and Mullen (J. E. C.) (1949). - Rapid and simple phosphatase test for milk. J. Dairy Res., 16, 58-67.

[14] PaschKe (B.) (1958). - Regeneration of phosphatase. Milchwissenschaft, 13, 553-554. 